\title{
Efeitos da reabilitação pulmonar associada ao treino de equilíbrio em pacientes com doença pulmonar obstrutiva crônica: revisão sistemática com metanálise
}

\author{
The effects of pulmonary rehabilitation associated with \\ balance training in patients with chronic obstructive \\ pulmonary disease: a systematic review with metanalysis
}

\author{
Adriana Paula Feitoza Pinto' 1 (1) \\ Clarissa Rebeca Leite de Oliveira Nunes ${ }^{2}$ (1) \\ Helen Barros de Oliveira Alencar ${ }^{3}$ (1) \\ Janaina Tavares Barreto Colasso 4 (1) \\ Guilherme Silva ${ }^{5}$ (1)
}

\begin{abstract}
${ }^{1-4}$ Centro Universitário Uniateneu (Fortaleza). Ceará, Brasil. adriana-feitoza@bol.com.br, leite_rebeca@hotmail.com, fisio.helenbarros@gmail.com, janainabarretoce@gmail.com ${ }^{5}$ Autor para correspondência. Universidade de Fortaleza (Fortaleza), Instituto Dr. José Frota (Fortaleza). Ceará, Brasil. guilhermepinheiro87@hotmail.com
\end{abstract}

\begin{abstract}
RESUMO | INTRODUÇÃO: O equilíbrio é um desfecho que vem sendo bastante estudado nos últimos anos em pacientes com doença pulmonar obstrutiva crônica (DPOC), porém ainda não se sabe ao certo os efeitos da reabilitação pulmonar (RP) associada a um treino de equilíbrio (TE). OBJETIVO: Avaliar os efeitos da reabilitação pulmonar (RP) associada ao treino de equilíbrio em pacientes com DPOC por meio de uma revisão sistemática com metanálise. MÉTODOS: Foi realizado um levantamento de artigos publicados em revistas indexadas entre os anos de 2013 e 2019 nas bases de dados: PUBMED, LILACS, SciELO e PEDro. As buscas foram conduzidas entre março e abril de 2020 e os descritores combinados foram: "pulmonary rehabilitation" OR "rehabilitation" $A N D$ "balance" $O R$ "falls" $O R$ "risk of falls" e suas respectivas traduções para o espanhol e português. RESULTADOS: Um total de 2052 estudos foram identificados, porém somente 4 cumpriram os critérios de elegibilidade. A pontuação da qualidade metodológica na escala PEDro variou entre 5 e 7 foi escores, o que demonstra um bom desenho metodológico. Dos 3 estudos selecionados, 2 realizaram treinamento de equilíbrio convencional (em solo envolvendo equilíbrio funcional, marcha e postura) e 1 utilizou equipamento específico (plataforma vibratória). O protocolo de intervenção teve duração entre 6 e 24 semanas, tempo médio diário entre 30 e 60 minutos e frequência semanal de 2 a 3 vezes. CONCLUSÃO: Os achados desta revisão sistemática com metanálise sugerem que a RP associada ao TE parece promover efeitos benéficos no equilíbrio funcional de pacientes com DPOC estáveis quando comparado a RP ambulatorial usual.
\end{abstract}

PALAVRAS-CHAVE: Doença pulmonar obstrutiva crônica. Equilíbrio postural. Risco de quedas. Reabilitação.
ABSTRACT | INTRODUCTION: balance is an outcome that has been extensively studied in recent years in patients with chronic obstructive pulmonary disease (COPD), but the effects of pulmonary rehabilitation (PR) associated with balance training (BT) are not yet clear. OBJECTIVE: to evaluate the effects of PR associated to BT in patients with COPD through a systematic review with meta-analysis. METHODS: it was performed a review of articles published in indexed journals between 2013 and 2019 in the databases PUBMED, PUBMED, LILACS, SciELO e PEdro. All searches were conducted between March and April 2020 and the descriptors combined were: "pulmonary rehabilitation" OR "rehabilitation" AND "balance" OR "falls" OR "risk of falls" and their respective translations into Spanish and Portuguese. RESULTS: A total of 2052 studies were identified, but only 3 articles met the eligibility criteria. The PEDro methodological quality scores varied between 5 and 7 scores, which demonstrates a good methodological design. Of the 3 selected studies, 2 performed conventional BT (on the ground involving functional balance, gait and posture) and 1 used specific equipment (vibrating platform). The intervention protocol lasted between 6 and 24 weeks, mean daily time between 30 and 60 minutes and frequency of 2 to 3 times a week. CONCLUSION: The findings of this systematic review with meta-analysis suggest that PR associated to BT seems to improve balance of patients with stable COPD when compared to usual PR.

KEYWORDS: Chronic obstructive pulmonary disease. Postural balance. Risk of falls. Rehabilitation. 


\section{Introdução}

A Doença Pulmonar Obstrutiva Crônica (DPOC) é uma enfermidade pulmonar que apresenta limitações do fluxo aéreo de forma progressiva. No decorrer da doença, os pacientes podem apresentar manifestações como inflamação sistêmica, comorbidades cardiovasculares, disfunção muscular periférica, perda de peso e alterações psicológicas ${ }^{1,2}$.

Estudos relatam que entre os anos de 2005 e 2016 houve um aumento de $24,2 \%$ das taxas de óbitos, confirmando que a DPOC é a segunda principal causa de mortalidade no mundo indo de encontro com estimativas de pesquisas anteriores onde se afirmava que esta enfermidade seria a terceira causa de morte somente no ano de 2030. Adicionalmente, o número de casos da doença e de suas consequências ainda são consideradas subestimadas devido o diagnóstico ser geralmente tardio e a enfermidade estar em estado avançado ${ }^{3-5}$.

Independente das modificações que aconteçam no pulmão, as alterações sistêmicas em pacientes com DPOC dificultam o prognóstico, pois acarreta em piora da qualidade de vida (QV), aumento dos sintomas de ansiedade e depressão, redução da tolerância ao exercício e inúmeras manifestações músculo esqueléticas como: fraqueza muscular generalizada, redução da mobilidade, desnutrição e desmineralização óssea que culminam em um importante déficit do equilíbrio e aumentando o risco de quedas ${ }^{6,7}$.

O risco de quedas é considerado um tema relevante na saúde pública, pois além de levar a mortalidade e a morbidade, também está relacionado com a funcionalidade global em indivíduos suscetíveis a déficit de equilíbrio. Adicionalmente este evento está associado a admissão precoce em instituições de cuidados prolongados, portanto, reduzir o risco de queda é um importante objetivo de saúde pública².

Dentro desse contexto o programa de reabilitação pulmonar (RP) é extremamente importante, pois é uma estratégia que envolve o treinamento físico, educação em saúde e auto manejo e proporciona melhoras importantes nas capacidades físicas e funcionais, além de melhora da QV e dos sintomas de ansiedade e depressão apresentando forte graus de recomendação científica ${ }^{8-12}$.
O objetivo desse estudo foi avaliar os efeitos da RP associada ao treino de equilíbrio em pacientes com DPOC por meio de uma revisão sistemática com metanálise.

\section{Método}

Esta revisão foi conduzida de acordo com as recomendações das diretrizes do Preferred Reporting Items for Systematic Reviews and Meta-Analyses ${ }^{13}$.

\section{Estratégias de busca}

Foi realizado um levantamento de artigos científicos publicados em revistas indexadas entre os anos de 2013 e 2020 nas seguintes bases de dados eletrônicas: PUBMED, Literatura Latino-Americana e do Caribe em Ciências da Saúde (LILACS) e na coleção Scientific Electronic Library Online (SciELO), PEDro a partir da pergunta PICO (população/intervenção/ controle/desfechos) que englobou: $\mathrm{P}=$ pacientes com DPOC estáveis; I= reabilitação pulmonar associada ao treino específico de equilíbrio; $\mathrm{C}=$ pacientes com DPOC que realizaram $\mathrm{RP}$ usual; $\mathrm{O}=$ desfechos relacionados ao equilíbrio funcional.

Todas as buscas foram conduzidas entre 01 de janeiro e 01 de março de 2020 e os descritores utilizados e combinados foram: "pulmonary rehabilitation" $O R$ "rehabilitation" AND "balance" OR "falls" $O R$ "risk of falls" e suas respectivas traduções para o espanhol e português. Tais termos foram conduzidos a partir de busca na lista do Medical Subject Headings (MeSH) do PUBMED e do DECS da Biblioteca Virtual em Saúde.

Os artigos foram selecionados por dois examinadores de forma independente a partir da análise do título, resumo e texto completo, respectivamente. Caso não houvesse consenso sobre a seleção de algum estudo, um terceiro autor realizou o julgamento final sobre a inclusão ou exclusão de artigos.

\section{Critérios de elegibilidade}

Foram incluídos no estudo artigos originais publicados no idioma inglês, espanhol ou português, do tipo ensaio clínico aleatorizado que envolviam adultos com DPOC estáveis submetidos a um programa RP ambulatorial usual com duração mínima de 6 semanas e que tenham avaliados como desfecho o equilíbrio 
utilizando como instrumento de avaliação a Escala de Equilíbrio de Berg (EEB) por se tratar do instrumentos mais utilizado na literatura. Estiveram excluídos desta pesquisa artigos indexados em mais de uma base de dados e estudos na qual os pacientes com DPOC apresentavam outras doenças respiratórias crônicas.

\section{Extração dos dados}

Um formulário padronizado foi usado para obter informações relevantes de artigos elegíveis que incluiu: autores, ano da publicação, país de origem, tamanho amostral, tempo/frequência/duração dos protocolos de intervenção, instrumentos utilizados para mensurar o equilíbrio e principais achados.

\section{Análise da qualidade metodológica dos estudos}

A qualidade metodológica dos estudos foi avaliada por meio da Escala de PEDro que é um instrumento válido e confiável composto de 11 itens que analisam o desenho metodológico e os resultados de estudos do tipo ensaio clínico sendo composta de 11 itens e avalia a descrição dos critérios de inclusão e exclusão, o cegamento de avaliadores, terapeutas e pacientes, a alocação dos sujeitos, a semelhança de indicadores de prognóstico entre os grupos, avaliação dos resultadoschave em pelo menos $85 \%$ dos sujeitos aleatorizados entre os grupos, análise dos resultados-chave por "intenção de tratamento" quando não foi possível receber o tratamento ou a condição controle conforme alocação e a descrição estatística de diferenças intergrupos ou medidas de variabilidade para pelo menos um resultado-chave ${ }^{14,15}$.

\section{Análise estatística dos dados}

Os dados foram analisados por meio do software da Cochrane (Review Manager - RevMan, versão 5.3). Os estudos elegíveis foram analisados utilizando-se a média e os desvio padrão (DP) para mensurar a mudança do baseline e o final de cada intervenção. Como todos os resultados foram variáveis contínuas, a diferença das médias (DM) e o intervalo de confiança de $95 \%$ (IC95\%) foram calculados quando os estudos descreveram em seus resultados das variáveis com as mesmas unidades de medida. Os resultados foram expostos em formato de gráficos de forest plot.

\section{Resultados}

Um total de 2052 estudos foram identificados a partir das bases de dados, porém somente 3 artigos cumpriram os critérios de elegibilidade da revisão (Figura 1).

A sumarização das características dos artigos selecionados no estudo, protocolos de intervenção, instrumentos utilizados, qualidade metodológica e principais achados encontra-se disponível na Tabela 1.

A pontuação da qualidade metodológica variou entre 5 e 7 escores na escala PEDro, o que demonstra um bom desenho metodológico em todos os estudos selecionados (Tabela 2).

Dos 3 estudos selecionados que avaliaram o impacto da RP no equilíbrio 2 realizaram treinamento de equilíbrio convencional (em solo envolvendo melhora do equilíbrio funcional, marcha e postura) e 1 utilizou equipamentos específico (plataforma vibratória).

O protocolo de intervenção teve duração entre 6 e 24 semanas, tempo médio diária entre 30 e 60 minutos e frequência de 2 a 3 vezes por semana.

No estudo de Beauchamp et al. ${ }^{16}$ verificou-se que os pacientes que realizaram um treinamento de equilíbrio associado a RP usual constituído de exercícios estáticos e dinâmicos em solo de transferência de postura, marcha e fortalecimento muscular funcional comparando-se com o grupo que recebeu somente a RP usual apresentou melhoras significativas nesse desfecho por meio da pontuação da EEB (diferença média: 5,4 escores; IC95\%:2,31 a 8,49; $p<0,01$ ) (Figura 1).

Os achados da pesquisa de Mkcher et al. ${ }^{17}$ evidenciaram que os pacientes que realizaram um treinamento de equilíbrio associado a RP usual constituído de exercícios de postura, transferências, exercícios de transição, marcha e fortalecimento muscular funcional quando comparado a um grupo que recebeu a RP usual apresentou melhoras significativas por meio da pontuação da EEB (diferença média: 7,30 escores; IC95\%: 6,66 a 7,94; $p<0,01)$. 
A pesquisa de Spielmanns et al. ${ }^{18}$ (2016) identificou que os pacientes que realizaram um treinamento de equilíbrio realizado em uma plataforma vibratória associada a RP usual quando comparado a um grupo que recebeu a RP usual apresentou melhoras similares na pontuação da EEB (diferença média: 1,5 escores; IC95\%: -1,03 a 4,03; $p=0,3$ ).

Figura 1. Fluxograma de seleção dos estudos "efeitos da RP associada ao treino de equilíbrio em pacientes com DPOC", 2013 a 2020

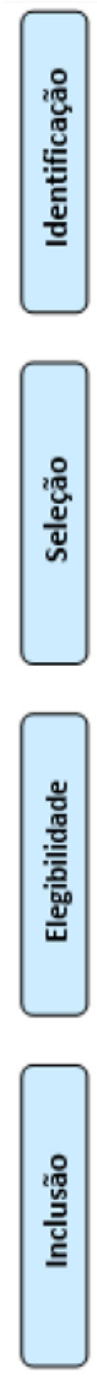

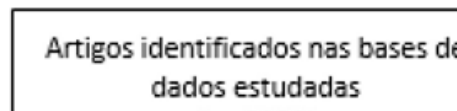
dados estudadas $(n=2052)$
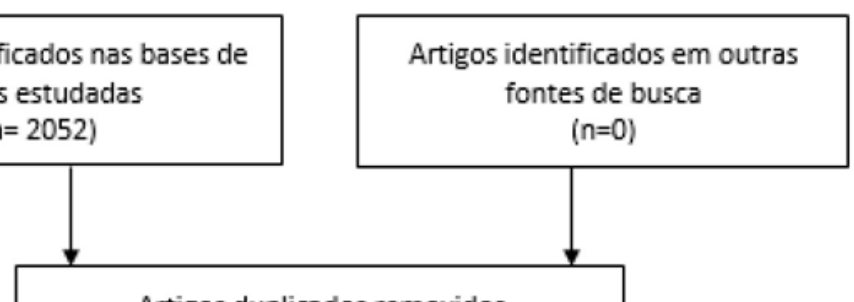

Artigos duplicados removidos

( $\mathrm{n}=1907$ )

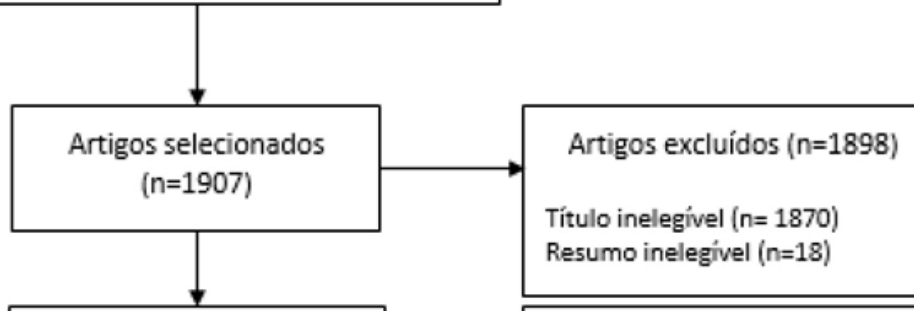

Texto completo excluídos

$$
(n=7)
$$

Não descreveu o protocolo de RP de forma completa $=2$ Protocolo de RP não envolveu sessões educacionais $=2$ Protocolo de RP com duração menor que 6 semanas $=1$

Não foram utilizados instrumentos para medição do equilibrio/risco de quedas $=2$

Figura 2. Comparação dos escores da Escala de equilíbrio de Berg em pacientes submetidos a Reabilitação pulmonar associado ao treino de equilíbrio versus reabilitação pulmonar ambulatorial usual

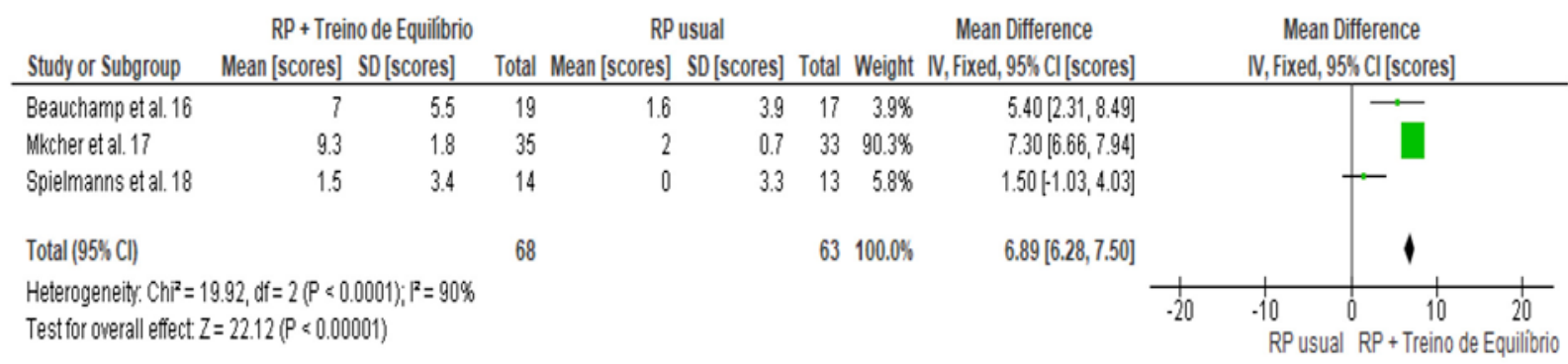


Tabela 1. Caracterização dos estudos selecionados na revisão sistemática descritos por autor/ano, país de origem, tamanho da amostra, protocolo de intervenção, qualidade metodológica e principais achados 2013 a 2020

\begin{tabular}{|c|c|c|c|c|c|}
\hline Autor/ano & País & Tamanho da amostra & $\begin{array}{l}\text { Protocolo de } \\
\text { intervenção }\end{array}$ & $\begin{array}{l}\text { Qualidade } \\
\text { metodológica }\end{array}$ & $\begin{array}{c}\text { Principais } \\
\text { achados } \\
\text { (diferença média } \\
\text { entre os grupos; } \\
\text { IC95\%) }\end{array}$ \\
\hline $\begin{array}{l}\text { Beauchamp et al., } \\
2013^{16}\end{array}$ & Canadá & $\begin{array}{c}39 \text { pacientes, } 21 \text { no GI (RP } \\
\text { usual + treinamento de } \\
\text { equilíbrio e } 18 \text { no GC (RP } \\
\text { usual) }\end{array}$ & $\begin{array}{c}6 \text { semanas, } \\
3 x / \text { semana, } 30 \text { min }\end{array}$ & 7 & $\begin{array}{c}\text { 5,4 escores; } \\
\text { IC95\%: 2,31 a 8,49; } \\
p<0,01\end{array}$ \\
\hline $\begin{array}{l}\text { Mkcher et al., } \\
2015^{17}\end{array}$ & Tunísia & $\begin{array}{c}68 \text { pacientes, } 35 \text { no GI (RP } \\
\text { usual + treinamento de } \\
\text { equilibrio e } 33 \text { no GC (RP } \\
\text { usual) }\end{array}$ & $\begin{array}{c}24 \text { semanas, } \\
3 x / \text { semana, } 30 \mathrm{~min}\end{array}$ & 5 & $\begin{array}{c}\text { 7,30 escores; } \\
\text { IC95\%: 6,66 a 7,94; } \\
p<0,01\end{array}$ \\
\hline $\begin{array}{l}\text { Spielmanns et al., } \\
2016^{18}\end{array}$ & Alemanha & $\begin{array}{l}29 \text { pacientes, } 14 \text { no GI } \\
\text { (treinamento de equilibrio) } \\
\text { e } 13 \text { no GC (RP usual) }\end{array}$ & $\begin{array}{c}12 \text { semanas, } \\
2 x / \text { semana, } 30 \text { min }\end{array}$ & 5 & $\begin{array}{c}\text { 1,5 escores; } \\
\text { IC95\%: }-1,03 \text { a } \\
\text { 4,03; } p=0,3\end{array}$ \\
\hline
\end{tabular}

$\mathrm{EEB}=$ Escala de equilíbrio de Berg; $\mathrm{DPOC}=$ doença pulmonar obstrutiva crônica;

$\mathrm{GC}=$ grupo controle; $\mathrm{GI}=$ grupo intervenção; $\mathrm{min}=$ minutos; $\mathrm{RP}=$ reabilitação pulmonar.

Tabela 2. Análise de qualidade metodológica dos estudos "efeitos da RP associada ao treino de equilíbrio em pacientes com DPOC", 2013 a 2020 selecionados por meio da Escala de PEDro

\begin{tabular}{|c|c|c|c|}
\hline Critérios da Escala PEDro & Beauchamp et al. ${ }^{16}$ & Mkcher et al. ${ }^{17}$ & Spielmanns et al. ${ }^{18}$ \\
\hline Critérios de inclusão selecionados & Sim & Não & Sim \\
\hline Alocação aleatória & Sim & Sim & Sim \\
\hline Sigilo na alocação & Sim & Não & Não \\
\hline Similaridade dos grupos no pré tratamento & Sim & Sim & $\operatorname{Sim}$ \\
\hline Cegamento dos sujeitos & Não & Não & Não \\
\hline Cegamento dos terapeutas & Não & Não & Não \\
\hline Cegamento dos examinadores & Sim & Não & Não \\
\hline Follow up de $85 \%$ dos participantes & Sim & Sim & Sim \\
\hline Intenção de tratar & Não & Não & Não \\
\hline Comparação estatística entre os grupos & Sim & Sim & Sim \\
\hline Relato das medidas de variabilidade & Sim & Sim & Sim \\
\hline Pontuação total & 7 & 5 & 5 \\
\hline
\end{tabular}

PEDro: Physiotherapy evidence database. 


\section{Discussão}

No nosso conhecimento ainda não existe na literatura vigente nenhum estudo de revisão sistemática com metanálise que tenha avaliado os efeitos da RP associada ao treino de equilíbrio em pacientes com DPOC, fato este que torna os resultados desta pesquisa relevantes, pois evidenciou-se que estes melhoram de forma significativa o equilíbrio funcional após participarem desta estratégia. Tal achado pode ser particularmente útil, pois poderá auxiliar no manejo clínico desta população, dar base para protocolos mais efetivos e prevenir possíveis complicações associadas.

A DPOC apresenta inúmeras manifestações pulmonares e extrapulmonares e está associada inúmeras comorbidades nas quais se incluem a doença cardiovascular, diabetes mellitus, osteoporose, depressão, fraqueza muscular, redução da mobilidade e consequente comprometimento do equilíbrio, sendo considerados preditores de quedas o que acarreta em redução importante na qualidade de vida desta população ${ }^{19}$.

O estudo de revisão sistemática de Hakamy et al. ${ }^{20}$ aponta que as evidências científicas a respeito dos efeitos da RP no equilíbrio e sobrevida são escassas e inconclusivas, porém nesta pesquisa os estudos identificados sobre a temática apresentaram resultados benéficos, apesar do pequeno tamanho amostral e pequenas diferenças de médias na EEB.

Estudos anteriores mostraram que o exercício reduz as quedas em idosos e que a RP usual quando associada a um componente de treinamento de equilíbrio, teve um efeito melhor no equilíbrio funcional e força muscular de pacientes com DPOC, o que pode culminar em redução das quedas ${ }^{21-23}$. Dessa forma, acreditamos que estudos de seguimento prospectivos seriam importantes para avaliar a curto, médio e longo prazo os efeitos da RP associada ao TE nessa população.

Seguindo essa linha de pensamento Verfasser ${ }^{24}$ ressalta que os exercícios com treino de equilíbrio apresentam moderado grau de recomendação e devem ser implementados para prevenção de quedas em idosos o que corrobora com os achados desta pesquisa, visto que a população DPOC é, majoritariamente, composta de indivíduos nesta faixa etária e foi possível verificar benefícios no grupo que recebeu tanto a RP associada ao treinamento de equilíbrio como a RP ambulatorial usual.
Dentro desse contexto ressalta-se a importância da participação de pacientes com DPOC em programas de RP, pois a literatura já está bem estabelecida quanto aos ganhos em aspectos físicos (tolerância ao exercício, capacidade funcional), mentais (sintomas de ansiedade e depressão), sociais (redução do isolamento social) e os achados desta pesquisa nos levam ao direcionamento que esta intervenção também resulta em melhora do equilíbrio, logo uma combinação de todas estes ganhos pode levar a uma possível redução de quedas ${ }^{12,25}$.

Estudo de coorte observacional verificou que a incidência de quedas em pacientes com DPOC é de 1,2 pessoas por ano, o que é 4 vezes maior que a incidência relatada em idosos ${ }^{26}$. Adicionalmente, outros estudos que avaliaram se o controle postural poderia discriminar indivíduos considerado como "caidores" e "não caidores" verificou que $46 \%$ dos participantes relataram pelo menos uma queda no ano anterior ${ }^{2,27}$.

No que se refere ao tempo de tratamento evidenciouse uma variedade importante no tempo de intervenção (6 a 24 semanas) o que vai de encontro com as evidências de estudos clássicos ${ }^{9,11,12}$ que afirmam que o período mínimo de duração de programas de RP é entre 6 e 12 semanas. O principal motivo da escolha do aumento desse período se deu após um estudo prévio do mesmo grupo ter verificado baixa eficácia do treinamento de equilíbrio associado a RP em pacientes com DPOC moderada a grave ${ }^{28}$. Além disso, esse mesmo estudo aponta que as diretrizes para prevenção de quedas recomendam treinamento contínuo em equilíbrio acima de 6 meses para alcançar resultados mais favoráveis na redução de quedas em idosos.

Os três estudos selecionados para a metanálise foram realizados no Canadá, Alemanha e Tunísia. Acreditamos que questões socioculturais podem influenciar nos resultados da intervenção, pois é necessário um suporte tecnológico e investimento financeiro para realização de protocolos de estudo que envolvam o treino de equilíbrio específico para pacientes com DPOC.

O único estudo que não apresentou resultado significativo na melhora do equilíbrio funcional foi o de Spielmanns et al. ${ }^{18}$. Acreditamos que isso pode ter ocorrido devido à baixa frequência semanal do protocolo de intervenção e porque os autores utilizaram 
apenas a plataforma vibratória como ferramenta para o treino de equilíbrio. Dentro desse contexto, seria importante que em estudos futuros fosse realizado a comparação de protocolos de treinamento associando ou não o uso da plataforma vibratória ao treino de exercícios em solo.

Esta pesquisa apresenta pontos fortes em seu desenho metodológico, porém apresenta limitações, visto que o número de artigos selecionados foi reduzido. Além disso utilizamos apenas um teste de avaliação de equilíbrio funcional (EEB) e os protocolos de RP usual e de treino de equilíbrio foram heterogêneos.

\section{Conclusão}

Os achados desta revisão sistemática com metanálise sugerem que a RP associada ao treino de equilíbrio promove efeitos benéficos no equilíbrio funcional de pacientes com DPOC estáveis quando comparado a RP ambulatorial usual.

\section{Contribuição dos autores}

Pinto APF, Nunes CBLO, Alencar HBO, Colasso JTB foram responsáveis por concepção e desenho da pesquisa, análise e interpretação dos dados, redação do manuscrito. Silva GPF foi responsável por concepção e desenho da pesquisa; análise e interpretação dos dados, redação do manuscrito e revisão crítica da versão final.

\section{Conflitos de interesses}

Nenhum conflito financeiro, legal ou político envolvendo terceiros (governo, empresas e fundações privadas, etc.) foi declarado para nenhum aspecto do trabalho submetido (incluindo, mas não se limitando a subvenções e financiamentos, participação em conselho consultivo, desenho de estudo, preparação de manuscrito, análise estatística, etc.).

\section{Referências}

1. Vogelmeier CF, Criner GJ, Martinez FJ, Anzueto A, Barnes PJ, Bourbeau J et al. Global strategy for the diagnosis, management, and prevention of chronic obstructive lung disease 2017 report: GOLD executive summary. Eur Respir J. 2071;195(5):557-582. doi: 10.1164/rccm.201701-0218PP
2. Crişan AF, Oancea C, Timar B, Fira-Mladinescu O, Tudorache V. Balance impairment in patients with COPD. PLoS One. 2015;10(3):e0120573. doi: 10.1371/journal.pone.0120573

3. GBD 2016 Risk Factors Collaborators. Global, regional, and national comparative risk assessment of 84 behavioural, environmental and occupational, and metabolic risks or clusters of risks, 1990-2016: a systematic analysis for the Global Burden of Disease Study 2016. Lancet. 2017;390(10100):1345-1422. doi: 10.1016/S0140-6736(17)32366-8. Erratum in: Lancet. 2017;390(10104):1736.

4. Lozano R, Naghavi M, Foreman K, Lim S, Shibuya K, Aboyans $\checkmark$ et al. Global and regional mortality from 235 causes of death for 20 age groups in 1990 and 2010: a systematic analysis for the global burden of disease study. 2010. Lancet. 2012;380(9859):2095-128. doi: 10.1016/S0140-6736(12)61728-0

5. Lopez AD, Shibuya K, Rao C, Mathers CD, Hansell AL, Held LS et al. Chronic obstructive pulmonary disease: Current burden and future projections. Eur Respir J. 2016;27(2):397-412. doi: $\underline{10.1183 / 09031936.06 .00025805}$

6. Paul T, Salazar-Degracia A, Peinado VI, Tura-Ceide O, Blanco I, Barreiro E et al. Soluble guanylate cyclase stimulation reduces oxidative stress in experimental Chronic Obstructive Pulmonary Disease. PLoS ONE. 2018;13(1):e0190628. doi: 10.1371/journal. pone. 0190628

7. Smith MD, Chang AT, Seale HE, Walsh JR, Hodges PW. Balance is impaired in people with chronic obstructive pulmonary disease. Gait Posture. 2010;31(4):456-60. doi: 10.1016/j. gaitpost.2010.01.022

8. Rinaldo N, Bacchi E, Coratella G, Vitale F, Milanesi C, Rossi A et al. Effects of Combined Aerobic-Strength Training vs Fitness Education Program in COPD Patients. Int J Sports Med. 2017;38(13):1001-1008. doi: 10.1055/s-0043-112339

9. Rochester, CL, Vogiatzis I, Holland AE, Lareau SC, Marciniuk DD, Puhan MA. An Official American Thoracic Society/European Respiratory Society Policy Statement: Enhancing Implementation, Use, and Delivery of Pulmonary Rehabilitation. Am J Respir Crit Care Med. 2015;192(11):1373-86. doi: 10.1164/rccm.2015101966ST

10. Corhay JL, Dang DN, Cauwenberge HV, Louis R. Pulmonary rehabilitation and COPD: providing patients a good environment for optimizing therapy. Int J Chron Obstruct Pulmon Dis. 2014;9:27-39. doi: 10.2147/COPD.S52012

11. Spruit MA, Pitta F, Garvey C, ZuWallack RL, Roberts CM, Collins EG et al. Differences in content and organizational aspects of pulmonary rehabilitation programmes. Eur Respir J. 2014;43(5):1326-37. doi: 10.1183/09031936.00145613 
12. Spruit MA, Singh SJ, Garvey C, ZuWallack R, Nici L, Rochester C et al. Na official American Thoracic Society/European Respiratory Society statement: key concepts and advances in pulmonary rehabilitation. Am J Respir Crit Care Med. 2013;188(8):13-64. doi: 10.1164/rccm.201309-1634ST

13. Moher D, Liberati A, Tetzlaff J, Altman DG. Preferred reporting items for systematic reviews and meta-analyses: The PRISMA statement. PLoS Med. 2009;6(7):e1000097. doi: 10.1371/journal. pmed.1000097

14. Moseley AM, Herbert RD, Sherrington C, Maher CG. Evidence for physiotherapy practice: a survey of the Physiotherapy Evidence Database (PEDro). Aust J Physiother. 2002;48(1):43-9. doi: 10.1016/s0004-9514(14)60281-6

15. Shiwa SR, Moseley AM, Maher CG, Pena Costa LO. Language of publication has a small influence on the quality of reports of controlled trials of physiotherapy interventions. J Clin Epidemiol. 2013;66(1):78-84. doi: 10.1016/j.jclinepi.2012.08.004

16. Beauchamp MK, Janaudis-Ferreira T, Parreira V, Romano JM, Woon L, Goldstein RS et al. A randomized controlled trial of balance training during pulmonary rehabilitation for individuals with COPD. Chest. 2013;144(6):1803-1810. doi: 10.1378/ chest.13-1093

17. Mkacher W, Mekki M, Tabka Z, Trabelsi Y. Effect of 6 Months of Balance Training During Pulmonary Rehabilitation in Patients With COPD. J Cardiopulm Rehabil Prev. 2015;35(3):207-13. doi: $10.1097 /$ $\underline{\text { HCR.0000000000000109 }}$

18. Spielmanns M, Boeselt T, Gloeckl R, Klutsch A, Fischer H, Polanski $\mathrm{H}$ et al. Low-Volume Whole-Body Vibration Training Improves Exercise Capacity in Subjects With Mild to Severe COPD. Respir Care. 2017;62(3):315-323. doi: 10.4187/respcare.05154

19. Barr RG, Celli BR, Mannino DM, Petty T, Rennard SI, Sciurba FC et al. Comorbidities, patient knowledge, and disease management in a national sample of patients with COPD. Am J Med.

2009;122(4):348-55. doi: 10.1016/j.amjmed.2008.09.042

20. Hakamy A, Bolton CE, McKeever TM. The effect of pulmonary rehabilitation on mortality, balance, and risk of fall in stable patients with chronic obstructive pulmonary disease. Chron Respir Dis. 2017;14(1):54-62. doi: 10.1177/1479972316661925

21. Harrison SL, Beauchamp MK, Sibley K, Araujo T, Romano R, Goldstein RS et al. Minimizing the evidence-practice gap - a prospective cohort study incorporating balance training into pulmonary rehabilitation for individuals with chronic obstructive pulmonary disease. BMC Pulm Med. 2015;15:73. doi: 10.1186/ s12890-015-0067-2

22. Marques A, Jácome C, Cruz J, Gabriel R, Figueiredo D. Effects of a pulmonary rehabilitation program with balance training on patients with COPD. J Cardiopulm Rehabil Prev. 2015;35(2):154-8. doi: $10.1097 /$ HCR. 0000000000000097
23. Gillespie LD, Robertson MC, Gillespie WJ, Sherrington C, Gates $\mathrm{S}$, Clemson LM et al. Interventions for preventing falls in older people living in the community. Cochrane Database Syst Rev. 2009;2:CD007146. doi: 10.1002/14651858.CD007146.pub3

24. Lundebjerg N. Guideline for the prevention of falls in older persons. American Geriatrics Society, British Geriatrics Society, and American academy of orthopaedic surgeons panel on falls prevention. J Am Geriatr Soc. 2001;49(5):664-72. doi: 10.1046/j.1532-5415.2001.49115.x

25. McCarthy B, Casey D, Devane D, Murphy K, Murphy E, Lacasse Y. Pulmonary rehabilitation for chronic obstructive pulmonary disease. Cochrane Database Syst Rev. 2015;(2):CD003793. doi: 10.1002/14651858.CD003793.pub3

26. Roig M, Eng JJ, Maclntyre DL, Road JD, FitzGerald JM, Burns J et al. Falls in people with chronic obstructive pulmonary disease: an observational cohort study. Respir Med. 2011;105(3):461-9. doi: 10.1016/j.rmed.2010.08.015

27. Beauchamp MK, Hill K, Goldstein RS, Janaudis-Ferreira T, Brooks D. Impairments in balance discriminate fallers from nonfallers in COPD. Respir Med. 2009;103(12):1885-91. doi: 10.1016/j. rmed.2009.06.008

28. Beauchamp MK, Janaudis-Ferreira T, Parreira V, Romano $J M$, Woon L, Goldstein RS et al. A randomized controlled trial of balance training during pulmonar rehabilitation for individuals with COPD. Chest. 2013;144(6):1803-1810. doi: 10.1378/ chest.13-1093 